Ann. Sci. forest., 1979, 36 (3), 211-229.

\title{
Une méthode de définition des stations en forêt : application à la forêt domaniale de Bellême
}

\author{
J. F. PICARD \\ Avec la collaboration de J. P. COURCHINOUX et Ch. KIEFFER \\ Laboratoire de Phytoécologie forestière, \\ Centre National de Recherches forestières, I.N.R.A., \\ Champenoux, 54280 Seichamps
}

\begin{abstract}
Résumé
Cet article propose une méthode de définition des stations forestières en utilisant l'exemple de la forêt domaniale de Bellême (Orne). Après une présentation du massif, on décrit la méthode utilisée.

- Sur le terrain : un premier passage au cours duquel seule la flore est relevée ; un deuxième passage dont l'objectif est de décrire, au moyen de relevés soigneusement choisis, les principaux types de stations préalablement définis ;

- Pour l'exploitation des résultats : elle procède en deux étapes, d'abord un premier tri au moyen de l'analyse factorielle des correspondances; ensuite l'élaboration d'un tableau phytosociologique où les stations sont caractérisées par diverses combinaisons originales d'un certain nombre de groupes sociologiques.
\end{abstract}

L'éfude a permis de mettre en évidence six principaux types de stations.

\section{Introduction}

Depuis quelques années, plus particulièrement depuis la fin des années 1960, on assiste à une généralisation de l'utilisation de méthodes d'analyse multivariable dans de nombreux domaines, biologiques ou non.

En matière d'étude de communautés végétales, plusieurs auteurs ( ${ }^{1}$ ) se sont penchés sur les nombreuses méthodes de classement ou d'ordination de ces communautés, comparant l'efficacité de ces méthodes ainsi que l'opportunité d'utiliser tel ou tel coefficient de similitude, d'aggrégation ou autre... A un point tel que Gauch et Whittaker (1972) ont pu écrire «One might say we need an ordination of ordination technics ». Par ailleurs, certains arrivent à des conclusions, au moins apparemment, contradictoires. Ainsi, Austin et Orloci (1966) ont dit «A comparison of the ordination results reveals a relatively clear separation of the major types by principal components analysis in contrast to the Bray-Curtis ordination, which promote an appearence of

(1) Parmi les auteurs ayant particulièrement travaillé ces méthodes, on peut citer Austin (1971), Austin et Orloci (1966), Orloci (1966-1975), Whittaker (1960-1967-1973), Curtis (1959), Grieg-Smith (1964), Swan (1966-1970), Mac Intosh (1967), Roux (1973), Noy-Meir (1974), Dagnelie (1960), et bien d'autres encore. Un excellent article de J. Esteve (1978) fait une sorte de synthèse critique des méthodes existantes. 
continuum 》 ou encore "A better method is principal component analysis of an appropriate similarity coefficient». Alors que, pour Gauch et Whittaker (1972) «The Bray-Curtis ordination using Sörensen's coefficient of community is found to be the best in general and principal component analysis the worst ».

La notion de continuum, par ailleurs très critiquée par Gounot (1969), est affirmée dans ce passage de Grant Cottam, Glenn Goff et Whittaker (1973) « The first (contribution of Wisconsin school) is in contributing to and stimulating research toward analysis of vegetation as a more or less continuous variable rather than as categorized types $»$.

En schématisant à l'extrême, on peut dire qu'aujourd'hui, deux courants s'opposent. Un premier, plus ancien, qui est classificatoire, peut être représenté par l'Ecole sigmatiste. Un deuxième, récent, qui est ordinatoire, peut être représenté par l'Ecole du Wisconsin.

Nous nous proposons ici d'exposer l'utilisation de l'analyse factorielle des correspondances (qui est un accomodement particulier de l'analyse en composantes principales proposé dans Benzecri (1973)) et la façon dont elle intervient dans la méthode d'étude des stations que nous proposons.

Cette méthode comporte trois phases que nous verrons plus en détail :

- une phase de terrain (prise des données) ;

- une phase d'analyse qui met en jeu l'analyse factorielle des correspondances ;

- une phase d'élaboration du résultat final qui fait intervenir un tableau phytosociologique.

\section{1. - Terrain}

\section{1. - Présentation du massif}

Cette méthodologie a été mise en œuvre pour l'étude de la Forêt domaniale de Bellême (Orne) dont nous faisons ci-dessous une présentation succinte.

Cette forêt ( $\left.{ }^{1}\right)$ s'étend sur environ 2500 ha, en un massif d'un seul tenant orienté Est-Ouest. Elle occupe un plateau d'altitude moyenne $200 \mathrm{~m}$ au relief varié mais peu accusé. Le substrat est composé pour les $2 / 3$ d'argile à silex, pour $1 / 3$ de sable du Perche, ces deux formations étant souvent recouvertes de limons d'épaisseur variable.

Le climat, de type atlantique, est tempéré, doux et humide. Orages, neige et grêle sont rares.

L'essence principale y est le Chêne sessile, le plus souvent associé au Hêtre : l'aménagement actuel a divisé la forêt en deux séries dont le découpage correspond, autant que faire se peut, aux beaux peuplements de Chêne sur limon pour la première, aux peuplements feuillus médiocres ou aux peuplements résineux pour la seconde.

\section{2. - Méthode d'inventaire}

Comme c'est le plus souvent le cas quand il s'agit de faire l'inventaire d'une surface d'un seul tenant, nous avons opté pour un échantillonnage systématique et au hasard.

$\left({ }^{1}\right)$ L'installation, en 1972, d'un important dispositif de recherches concertées sur la régénération du Chêne fut à l'origine du choix de cette forêt. 
Il a été obtenu en disposant sur un plan au 1/10 000 de la forêt une grille à maille carrée de 3,5 cm de côté (1 point pour 9 ha environ); cent quatre-vingt-douze points ont ainsi été repérés, à l'emplacement desquels un relevé phytosociologique (méthode zuricho-montpelliéraine) a été effectué.

Dans un premier temps, et comme nous l'avions préconisé à l'issue de notre travail sur les forêts de l'étage du Rhétien (Picard, 1970), nous avons limité nos observations à la seule flore. Ce n'est que lorsque les unités floristiques ont été connues que nous sommes revenu à l'emplacement des relevés caractéristiques pour y faire les descriptions de profil de sol et vérifier l'homogénéité des unités floristiques.

\section{2. - L'analyse des correspondances}

Nous avons utilisé, dans un premier temps, la méthode d'analyse factorielle des correspondances. Cette méthode, qui commence à être bien connue, ne sera pas décrite ici et nous renvoyons le lecteur, pour plus amples informations, à la bibliographie (entr'autres : Benzecri, 1973 ; Romane, 1972 ; Bachacou, 1975 ; Lacoste et Roux, 1971-1972 ; Kshirsagar, 1972). Précisons cependant que nous avons travaillé sur une matrice constituée par les coefficients d'abondance-dominance comportant cent soixante-dix-sept lignes (les relevés) et cent soixante-dix colonnes (les espèces). Parce qu'au moment du traitement informatique, nous ne disposions pas d'un ordinateur assez puissant, nous avons classé soixante-dix variables comme principales (elles participent à l'élaboration des axes factoriels) et cent comme supplémentaires (on les replace après coup dans l'espace factoriel).

\section{1. - Importance du $\operatorname{Cos}^{2}$}

L'analyse a été poussée jusqu'à l'obtention de cinq axes factoriels (ou « axes floristiques » ou "facteurs») dont nous avons pu interpréter les quatres premiers. Ces axes sont caractérisés par des espèces qui leur sont fortement liées soit du côté positif, soit du côté négatif. Trois éléments caractérisent chaque point :

- ses coordonnées,

- la qualité de la représentation (mesurée par le $\operatorname{Cos}^{2}$ ) ${ }^{1}$ ),

- la contribution.

En ce qui concerne plus particulièrement le $\mathrm{Cos}^{2}$, on comprend facilement que plus il est élevé, plus l'espèce en question est proche, dans l'espace, de l'axe ; et donc, plus sa position sur l'axe peut être considérée comme caractéristique de cet axe. Les différents axes étant en effet orthogonaux entre eux, un point dont le $\operatorname{Cos}^{2}$ est nul sur, par exemple, l'axe 1, est très éloigné de cet axe et n'en sera pas caractéristique. Par contre, il peut l'être d'un autre axe, d'ordre plus élevé. En poussant ce raisonnement à la limite, une espèce que nous supposerons avoir la plus forte valeur positive sur un axe mais dont le $\operatorname{Cos}^{2}$ serait faible, n'aurait qu'un faible caractère indicateur vis-à-vis de cet axe. On lui préfèrera telle autre espèce à abcisse peut-être moins élevée mais à fort $\operatorname{Cos}^{2}$.

(1) On a choisi le $\operatorname{Cos}^{2}$ pour plus de commodité, celui-ci étant toujours positif. 


\section{2. - Les axes factoriels}

L'analyse a permis la mise en évidence d'un certain nombre d'espèces suivant leur position sur les axes et le $\operatorname{Cos}^{2}$ qui leur est affecté. Sur les cent soixante-dix espèces rencontrées, soixante-treize (soit un peu moins de la moitié) sont sorties ( ${ }^{1}$ ) à l'analyse. Celles qui n'ont pas été retenues étaient le plus souvent (ou semblaient) ubiquistes ou, au contraire, rares (trois ou quatre présences sur l'ensemble du massif).

\subsection{Premier axe; valeur propre: 0,401 .}

II explique 13 p. 100 de la variation totale. Du côté positif, on trouve les espèces de limons à limons argileux, bien alimentés en eau (réserves importantes, fonction surtout de l'épaisseur de ces limons), peu acides et relativement riches (par rapport aux sables du Perche). Parmis les espèces dont le $\operatorname{Cos}^{2}$ est le plus faible, certaines ont une tendance à la calciphilie, comme le Brachypode des bois et l'Eglantier. Les peuplements, le plus souvent très fermés, sont plus riches en! Hêtre que,dans la moyenne de la forêt.

Cette composante semble ainsi tenir compte à la fois - et ces facteurs sont plus ou moins liés - du pH, de l'alimentation en eau, de la richesse chimique du sol.

\subsection{Deuxième axe ; valeur propre 0,246 .}

Il participe pour 8 p. 100 à la variation totale. Bien que les graphiques $1 \times 2$ présentent (fig. 1 et 2 ) ( ${ }^{2}$ ) un certain effet Guttman (Bachacou, 1973), nous avons pu lui donner une interprétation biologique. Les espèces de la « futaie (Chêne sessile ou Pin sylvestre) super acidophile ", normalement très ouverte, s'y trouvent opposées aux espèces de la «futaie acidophile » toujours très fermée. Ecologiquement parlant, il est manifeste que les espèces de la «futaie super-acidophile » sont également héliophiles et qu'à l'inverse, les espèces de la futaie acidophile sont sciatolérantes. La lumière apparaît comme le facteur écologique qui explique le mieux la distribution des espèces sur cet axe.

\subsection{Troisième axe ; valeur propre : 0,184.}

II participe encore pour 6 p. 100 à la variation totale. Du côté négatif, on retrouve certaines des espèces les plus caractéristiques de l'axe 1 (futaie Chêne-Hêtre fermée sur limon argileux) alors que du côté positif apparaissent des espèces héliophiles ou hélio-hygrophiles de sols limoneux à limono-sableux. Mais, alors que pour l'axe $n^{0} 2$ le facteur lumière apparaît comme lié à une structure naturelle du peuplement, une structure climacique, pour l'axe $n^{0} 3$, il apparaît comme une conséquence de l'intervention de l'homme. Les espèces caractéristiques de cet axe, côté positif, sont des espèces qu'on trouve en général dans les peuplements où tout ou partie du couvert forestier a été supprimé.

Pour nous résumer: bien que les axes 2 et 3 soient analogues quant au facteur

(1) On dit qu'une espèce est « sortie » à l'analyse quand son $\operatorname{Cos}^{2}$ est supérieur à 0,1 (valeur fixée plus ou moins arbitrairement) sur un axe au moins. Le tableau 1 en donne la liste.

$\left({ }^{2}\right)$ Les raisons du regroupement des espèces par groupe et des relevés par type sont exposées au paragraphe 3.2. 


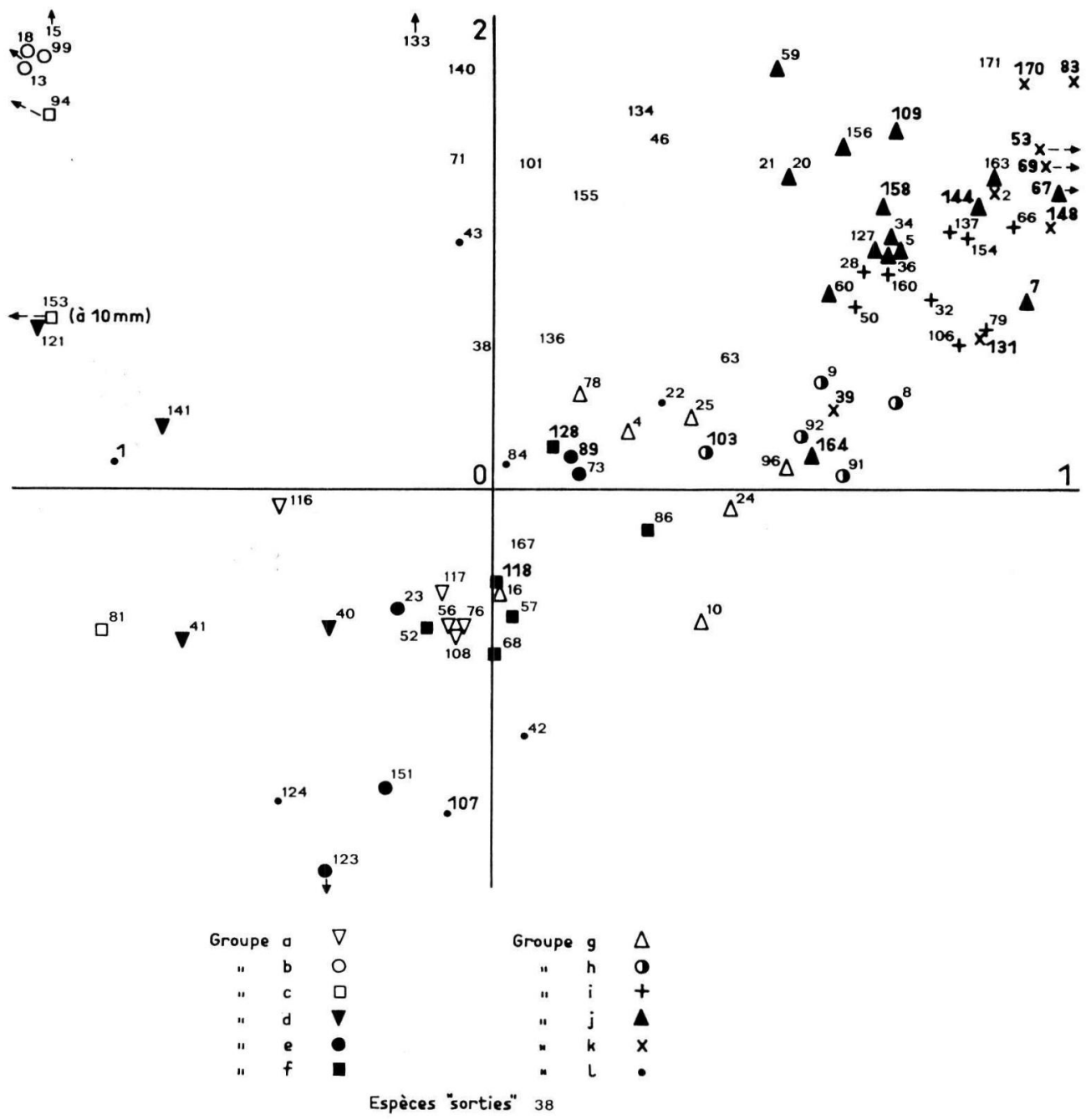

FIG. 1. - Répartition des espèces dans le plan factoriel $1 \times 2$.

Species disposition through factorial plan $1 \times 2$.

qu'ils mettent en évidence — la lumière - ils diffèrent nettement quant à l'origine de cette lumière qui est un élément permanent de la station pour l'axe 2 et un élément accidentel (et artificiel) pour l'axe 3.

2.24. Quatrième axe; valeur propre: 0,119 .

II ne participe plus que pour 4 p. 100 à la variation totale. Du côté négatif, on trouve des espèces soit nettement calcicoles, soit neutrophiles, mais restant le plus souvent abondantes sur terrain calcaire (comme le Coudrier). Du côté positif, les espèces caractéristiques sont trop peu nombreuses pour permettre une interprétation écologique valable. Notons que deux d'entr'elles participent également à l'axe 1.

II semble bien que l'on puisse interpréter cet axe comme opposant les sols calcaires aux sols acides, mais sans que nous puissions en avoir la certitude. En effet, 


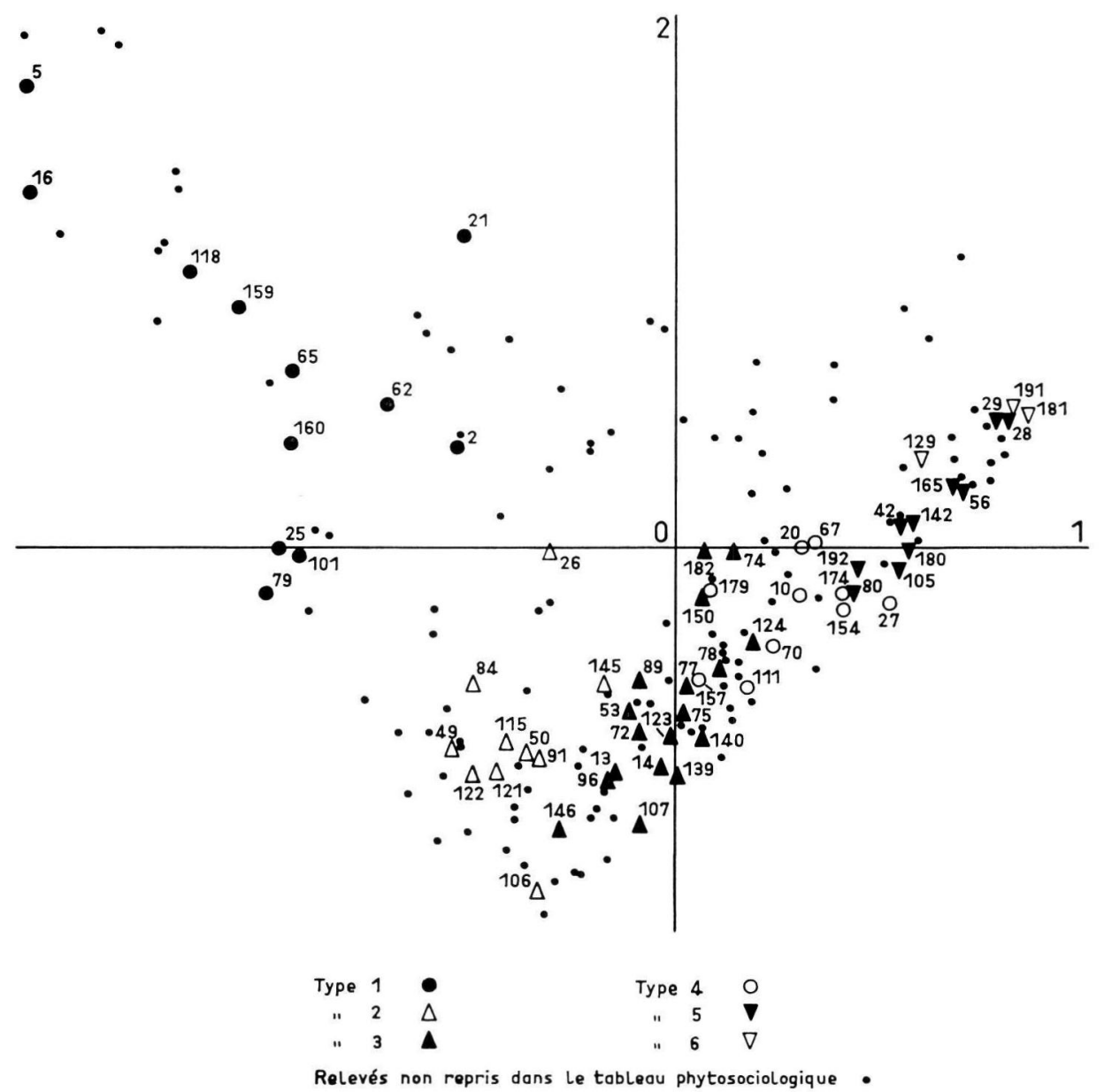

FIG. 2. - Répartition des relevés dans le plan $1 \times 2$.

Species lists disposition through factorial plan $1 \times 2$.

et c'est l'inconvénient majeur de la présélection qui a conduit à distinguer variables principales et supplémentaires, les premières sont utilisées pour l'élaboration des axes factoriels, les autres y sont simplement replacées après coup. S'il manque une dimension à l'espace défini par les variables principales, les espèces supplémentaires qui auraient pu se regrouper selon cette dimension ne peuvent se singulariser.

Dans l'exemple qui nous intéresse, la plupart des espèces calcicoles sont des variables supplémentaires : la dimension « calcaire » n'est donc qu'ébauchée dans l'espace des composantes. Pour confirmer (ou infirmer...) cette interprétation, nous avons fait récemment, et sur les mêmes données, une nouvelle analyse pour laquelle nous avons pu augmenter le nombre des variables principales (et y incorporer les espèces calcicoles). Les deux premiers axes factoriels obtenus sont identiques, quant à leur interprétation, aux deux premiers de la première analyse. Mais le facteur " calcaire » étant très caractérisé dans cette nouvelle analyse, il apparaît dès le troisième axe. 


\subsection{Cinquième axe ; valeur propre : 0,108.}

Participant pour 3 p. 100 à la variation totale, cet axe, à cause surtout du nombre restreint d'espèces le caractérisant, est difficile à interpréter. II semblerait que, du côté positif, l'on soit en présence d'espèces liées à la présence de limons riches (dans le contexte de Bellême), à humidité relativement élevée. Du côté négatif, il s'agit plutôt d'espèces, toujours liées à la présence de limons riches, mais de sol peu humides.

Malgré son numéro d'ordre élevé et sa valeur propre faible, nous avons cherché à interpréter cet axe parce qu'il met en valeur des espèces à écologie un peu particulière que nous retrouverons plus loin comme différentielles d'un faciès stationnel.

\section{3. - Essai de synthèse}

Les axes factoriels, élaborés à partir des variables floristiques, ont été interprétés grâce à ces variables.

L'analyse des correspondances nous a permis :

- de définir les principaux grandients écologiques existant dans la forêt étudiée et d'apprécier leur importance relative (ici, et comme souvent, c'est l'axe 1 qui est le plus important) ;

- de faciliter le choix (guidé par la valeur du $\operatorname{Cos}^{2}$ sur les axes) et l'ordre des espèces et des relevés devant constituer le tableau phytosociologique présenté cidessous ;

— d'ordonner espèces et relevés en fonction de ces gradients (fig. 1 et 2).

\section{3. - Le tableau phytosociologique}

\section{1. - Construction du tableau}

C'est un ensemble de relevés (disposés en colonnes) et d'espèces (disposées en lignes) que l'on cherche à ordonner de telle sorte qu'en fin de travail on obtienne un tableau dit « diagonalisé » (Guinochet, 1973, décrit en détail les façons de construire un tel tableau et les buts poursuivis). Cette diagonalisation est due au fait qu'espèces et relevés sont ordonnés selon le gradient écologique principal.

Pour rendre le tableau final plus simple et plus clair, plutôt que d'y faire figurer l'ensemble des cent soixante-dix-sept relevés, nous avons préféré choisir un échantillonnage restreint, mais qui reste représentatif de toute la variabilité. Pour cela, nous avons utilisé le graphique de la figure 2 (où figurent, accompagnés d'un symbole, les relevés qui apparaissent dans le tableau) en utilisant les critères de choix suivant :

- l'axe représentant le gradient principal étant l'axe 1, nous avons, dans un premier temps, choisi des relevés régulièrement répartis le long de cet axe. Pour les extrêmes, nous avons tenu compte des $\operatorname{Cos}^{2}$, choisissant de préférence les relevés à $\mathrm{Cos}^{2}$ élevé ;

- l'axe 3 caractérisant une héliophilie d'origine artificielle, donc des peuplements où la végétation risque d'avoir été perturbée, nous avons évité de choisir des relevés à fort $\operatorname{Cos}^{2}$ (et abcisse positive) sur ces axes ;

- enfin, nous avons choisi quelques-uns des relevés présentant sur les axes 4 et 5 un fort $\operatorname{Cos}^{2}$ (situés, sur l'axe 1, au voisinage du 0 ). 


\section{TABLEAU 1}

Espèces dont le $\operatorname{Cos}^{2}$ est supérieur à 100. Valeur de ce $\operatorname{Cos}^{2}$ sur les différents axes abscisse positive - abscisse négative

Valeur du $\operatorname{Cos}^{2} \times 1000$ sur les axes

Species for wich $\operatorname{Cos}^{2}$ is higher than 100. Value of $\operatorname{Cos}^{2}$ on different axes positive abcissa - negative abscissa

Value of $\operatorname{Cos}^{2} \times 1000$ on axes

\begin{tabular}{|c|c|c|c|c|c|c|}
\hline $\begin{array}{l}N^{0} \\
\text { code }\end{array}$ & Nom des plantes & Axe 1 & Axe 2 & Axe 3 & Axe 4 & Axe 5 \\
\hline 2 & Acer campestre $\ldots \ldots \ldots \ldots \ldots$ & & & & 175 & \\
\hline 4 & 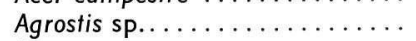 & & & 165 & & \\
\hline 5 & Ajuga reptans .............. & 226 & & & & \\
\hline 8 & Asperula odorata ............... & 367 & & 151 & & \\
\hline 9 & Athyrium filix femina.......... & 150 & & & & \\
\hline 10 & Atrichum undulatum $\ldots \ldots \ldots \ldots$ & & & & 133 & 136 \\
\hline 13 & Betula pubescens $(\mathrm{a}+\mathrm{g}) \ldots \ldots$ & 228 & 193 & & & \\
\hline 15 & Befula verrucosa $(a+g) \ldots \ldots$ & & 142 & & & \\
\hline 16 & Blechnum spicant . ............. & & & & & 122 \\
\hline 163 & Brachypodium silvaticum ........ & 116 & & & 102 & \\
\hline 18 & Calluna vulgaris................ & 360 & 316 & & & \\
\hline 20 & Carex glauca ................. & & & & 133 & \\
\hline 21 & Carex maxima $\ldots \ldots \ldots \ldots \ldots$ & & 110 & & & \\
\hline 22 & Carex pallescens ............. & & & & & 212 \\
\hline 23 & Carex pilulifera $\ldots \ldots \ldots \ldots \ldots$ & & & 114 & & \\
\hline 24 & Carex remota ............... & 119 & & & & \\
\hline 25 & Carex silvatica $\ldots \ldots \ldots \ldots \ldots$ & 137 & & & & \\
\hline 28 & Carpinus betulus (a) $\ldots \ldots \ldots \ldots$ & 112 & & & & \\
\hline 32 & Circaea lutetiana .............. & 348 & & 105 & & \\
\hline 34 & Corylus avellana $(\mathrm{a}+\mathrm{g}) \ldots \ldots$ & & & & 160 & \\
\hline 36 & Crataegus oxyacantha .......... & & & & 118 & \\
\hline 38 & Dactylis glomerata ........... & & & 108 & & \\
\hline 40 & Deschampsia flexuosa . . . . . . . . . & 180 & 133 & & & \\
\hline 41 & Dicranum scoparium $\ldots \ldots \ldots \ldots$ & 358 & & & & \\
\hline 42 & Dicranella heferomala $\ldots \ldots \ldots \ldots$ & & 121 & & 210 & \\
\hline 43 & Digitalis purpurea $\ldots \ldots \ldots \ldots$ & & 100 & 230 & & 142 \\
\hline 46 & Epilobium montanum .......... & & 153 & 174 & & \\
\hline 50 & Euphorbia amygdaloides...$\ldots \ldots$ & 332 & & & & \\
\hline 52 & Eurhynchium striatum ........ & & 158 & & & \\
\hline 56 & Fagus silvatica $(\mathrm{A}+\mathrm{a}) \ldots \ldots \ldots$ & & 312 & & & \\
\hline 57 & Fagus silvatica $(\mathrm{g}) \ldots \ldots \ldots \ldots$ & & 159 & & & \\
\hline 59 & Fragaria vesca ................ & & 108 & & & \\
\hline 60 & Fraxinus excelsior ............ & 111 & & & 119 & \\
\hline 63 & Galeopsis tetrahit . . . . . . . . . & & & & & 101 \\
\hline 66 & Geranium robertianum .......... & 118 & & & & \\
\hline 68 & Hedera helix .............. & & 474 & & & \\
\hline 71 & Holcus lanatus..$\ldots \ldots \ldots \ldots$ & & 115 & 386 & & \\
\hline 73 & Hypericum pulchrum ......... & & & 119 & & \\
\hline 76 & Ilex aquifolium................ & & 328 & & & \\
\hline 78 & Juncus sp. $\ldots \ldots \ldots \ldots \ldots \ldots$ & & & 296 & & \\
\hline 79 & Lamium galeobdolon ............. & 222 & & 135 & & \\
\hline 81 & Leucobryum glaucum .......... & 299 & & & & \\
\hline 84 & Luzula campestris . ............. & & & 123 & & \\
\hline 86 & Lonicera periclymenum ......... & 117 & & & & \\
\hline 91 & Melica uniflora $\ldots \ldots \ldots \ldots \ldots$ & 280 & & 123 & & \\
\hline 92 & Milium effusum . ............... & 257 & & & 103 & 135 \\
\hline 94 & Molinia coerulea $\ldots \ldots \ldots \ldots \ldots$ & 424 & 240 & & & \\
\hline 96 & Oxalis acetosella ................ & 212 & & & 100 & 100 \\
\hline 99 & Pinus silvestris . . . . . . . . . . . & 448 & 319 & & & \\
\hline
\end{tabular}


TABLEAU 1 (bis)

\begin{tabular}{|c|c|c|c|c|c|c|}
\hline $\begin{array}{c}\mathrm{N}^{\circ} \\
\text { code }\end{array}$ & Nom des plantes & Axe 1 & Axe 2 & Axe 3 & Axe 4 & Axe 5 \\
\hline 101 & Poa annua $\ldots \ldots \ldots \ldots \ldots \ldots$ & & & 278 & & \\
\hline 106 & Polystichum filix-mas.......... & 151 & & & & \\
\hline 108 & Polytrichum formosum ......... & & 388 & & & \\
\hline 171 & Prunus spinosa $\ldots \ldots \ldots \ldots \ldots$ & & & & 103 & \\
\hline 116 & Pteridium aquilinum .......... & 340 & & & & \\
\hline 117 & Quercus sessiliflora ........... & & 210 & & & \\
\hline 121 & Rhamnus frangula .......... & 317 & & & & \\
\hline 123 & Rhytidiadelphus loreus $\ldots \ldots \ldots$. & & 124 & & & \\
\hline 124 & Rhytidiadelphus triqueter........ & & 171 & & & \\
\hline 127 & Ros $a$ sp. $\ldots \ldots \ldots \ldots \ldots \ldots$ & 114 & & & & \\
\hline 133 & Salix caprea (a) _.......... & & 108 & 106 & & \\
\hline 134 & Salix caprea $(\mathrm{g}) \ldots \ldots \ldots \ldots$ & & & 168 & & \\
\hline 136 & Sarothamnus scoparius ......... & & & 161 & & \\
\hline 137 & Scrofularia nodosa $\ldots \ldots \ldots \ldots$ & 167 & & & & \\
\hline 140 & Sonchus sp................ & & & 140 & & \\
\hline 141 & Sorbus aucuparia $\ldots \ldots \ldots \ldots$ & 292 & & & & \\
\hline 167 & Sorbus torminalis ............ & & & & 154 & \\
\hline 151 & Thuidium tamariscifolium ...... & & 299 & & & \\
\hline 153 & Vaccinium myrtillus ............ & 551 & & & & \\
\hline 154 & Veronica montana .............. & 259 & & & & \\
\hline 155 & Veronica officinalis .......... & & & 198 & & \\
\hline 157 & Viburnum opulus ............. & & & & 120 & \\
\hline 160 & Viola silvestris $\ldots \ldots \ldots \ldots \ldots$ & 230 & & & & \\
\hline
\end{tabular}

En ce qui concerne les espèces, aucun choix n'a été fait : il nous a été imposé par la composition floristique des relevés. Nous avons été guidé dans leur ordination par leur position sur l'axe 1.

La première ordination a conduit à un premier tableau, relativement satisfaisant dans l'ensemble, que nous avons cependant dû réarranger à deux reprises (ce qui est peu) avant d'obtenir le tableau définitif (tabl. 2). Les critères obtenus lors de ces réarrangements successifs sont à la fois objectifs et subjectifs.

- « objectifs» parce que nous avons cherché autant que possible des résultats de l'analyse des correspondances autres que ceux obtenus à partir de l'axe 1, en particulier ceux des axes 4 et 5 (par exemple, singularisation d'espèces comme Blechnum spicant et Atrichum undulatum) ;

- « subjectifs », parce que nous avons utilisé des critères de classement que l'analyse ne peut prendre en compte et qui relèvent des connaissances a priori que l'on peut avoir de l'écologie des espèces. En particulier, on peut tenir compte non plus de la présence ou de l'abondance d'une espèce mais de son absence ou encore de la dissymétrie de sa distribution par rapport à l'ensemble des relevés : cela a été le cas, par exemple, pour Pteridium aquilinum et Deschampsia flexuosa.

Une fois que l'on considère l'ordination des espèces et des relevés comme optimale, on regroupe les espèces « caractéristiques » en groupes, la présence d'un ou plusieurs groupes servant à la définition, au niveau des relevés, des types de station.

Terminons en insistant sur le fait qu'il n'existe pas, pour un tableau, un seul arrangement optimal des espèces et des relevés. En particulier, au niveau des espèces 
peu fréquentes, on peut être tenté, suivant ce que l'on a comme connaissances a priori sur leur écologie et leur fréquence, de les ranger dans un groupe ou parmi les compagnes.

Dans notre cas, Scrofularia nodosa a d'abord été classée parmi les compagnes en raison de sa fréquence peu élevée : en définitive, à cause en partie de son $\operatorname{Cos}^{2}$ élevé sur l'axe 1, nous l'avons incorporée au groupe d'espèces dont elle était proche.

\section{2. - Interprétation du tableau}

Les différents groupes qui apparaissent dans le tableau phytosociologique sont interprétables écologiquement.

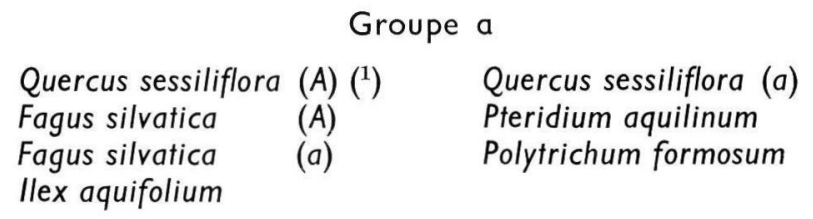

On y rencontre les espèces les plus constantes de la chênaie-hêtraie acidophile du bassin parisien. Elles ne sont caractéristiques d'aucun groupement particulier. Remarquons tout de même que la Fougère Aigle est beaucoup plus abondante dans les unité du type 1 et 2 que dans les autres.

\section{Groube b}

Betula pubescens $\quad$ Calluna vulgaris
Pinus silvestris

Différentiel du type 1. Les espèces qui le composent sont des super acidophiles héliophiles.

\section{Grouge c}

Molinia coerulea $\quad$ Leucobryum glaucum
Vaccinium myrtillus

ifférentiel du type 2. Ce sont des espèces acidophiles dont deux sont héliophiles. Remarquons que le développement de la Molinie, comme celui de la Myrtille, est maximum dans le type 1 .

\section{Groupe d}

$\begin{array}{ll}\text { Deschampsia flexuosa } & \text { Sorbus aucuparia } \\ \text { Dicranum scoparium } & \text { Rhamnus frangula }\end{array}$

Constant dans les types 1 et 2 , ce groupe se trouve également à la charnière des types 2 et 3 . Dans les unités du type 3, on rencontre encore certaines de ces espèces, mais rares et le plus souvent peu abondantes. II s'agit d'acidoclines banales.

( $\left.{ }^{1}\right) \mathrm{A}=$ Arbre.

$a=$ arbuste.

$g=$ germination ou jeune plant. 


\section{Groupe e}

Carex pilulifera

Hypericum pulchrum

Rhytidiadelphus loreus
Thuidium tamariscifolium

Luzula pilosa

Il s'agit également d'acidoclines, un peu moins affirmées que les précédentes. Ce groupe est caractéristique des types 2 et 3.

\section{Groupe $f$}

$\begin{array}{lll}\text { Quercus sessiliflora }(g) & \text { Rubus sp. } \\ \text { Fagus silvatica } & (g) & \text { Lonicera periclymenum } \\ \text { Hedera helix } & & \text { Eurhynchium striatum }\end{array}$

Avec ce groupe, on rencontre les premières espèces méso-acidophiles. Pour la plupart d'entre elles, ce sont également des sciatolérantes. Ce groupe caractérise en fait la chênaie sessiliflore et est présent à Bellême pratiquement partout. Normalement absent du type 1, on l'y rencontre parfois : il marque alors la transition avec le type 2. Remarquons également que dans le type 6 , le Chêne pédonculé a tendance à se substituer au Chêne sessile.

$\begin{array}{ll} & \text { Groupe g } \\ \text { Blechnum spicant } & \text { Carex remota } \\ \text { Juncus sp. } & \text { Carex silvatica } \\ \text { Agrostis sp. } & \text { Oxalis acetosella } \\ \text { Atrichum undulatum } & \end{array}$

Nous n'avons pas voulu individualiser d'unité spéciale au moyen de ce groupe parce que, sur le terrain, il n'occupe jamais des surfaces très importantes. Le plus souvent, ces espèces sont localisées à des secteurs bien particuliers : un peu plus clairs et surtout plus humides. Nous en avons fait un faciès du type $n^{\text {e }} 3$ parce que c'est là que ce groupe est le mieux individualisé.

\section{Groupe h}

$\begin{array}{ll}\text { Polygonatum multiflorum } & \text { Asperula odorata } \\ \text { Milium effusum } & \text { Athyrium filix femina } \\ \text { Melica uniflora } & \end{array}$

Composé de sciatolérantes et de meso-acidophiles typiques, l'apparition de ce groupe indique le passage du type 3 au type 4. Il différencie nettement des types 4, 5 et 6 des types 1, 2 et 3 où ces espèces n'apparaissent pratiquement jamais.

\section{Groupe i}

Veronica montana
Circaea lutetiana
Euphorbia amygdaloïdes
Polystichum filix-mas
Scrofularia nodosa

Annales des Sciences forestières. - 1979
Viola silvestris

Lamium galeobdolon

Carpinus betulus

Geranium robertianum 
II rassemble des espèces d'humus doux (mull mésotrophe). Ce sont des sciatolérantes, plus ou moins basoclines. L'espèce la plus fidèle au groupe est le Lamier jaune, la Circée et l'Euphorbe marquant plutôt la transition avec le type précédent.

\section{Groupe j}

Ajuga reptans

Vicia sepium

Rosa sp.

Fraxinus excelsior

Crataegus (2 sp.)

Brachypodium silvaticum

Deschampsia coespitosa

Viburnum opulus ( $\mathrm{a}$ et $\mathrm{g}$ )
Corylus avellana (a et $\mathrm{g}$ )

Carex glauca

Fragaria vesca

Potentilla fragariastrum

Geum urbanum

Stachys silvaticus

Arum maculatum

On y rencontre les premières basoclines typiques. Ce groupe fait transition entre les groupes i et $k$.

Groupe k
Quercus pedunculata
Daphne laureola
Acer campestre
Cornus sanguinea

Ligustrum vulgare

Evonymus vulgaris

Tamus communis

Helleborus fœidus

Ruscus aculeatus

II rassemble les calcicoles strictes, sauf peut-être pour le Fragon qui se trouve dans ce groupe du fait de sa localisation aux lisières exposées au sud qui sont toutes (ou presque) sur calcaire.

\section{Groupe I}
Abies alba
Dicranella heteromala
Digitalis purpurea

\begin{abstract}
Rhytidiadelphus triqueter
Carex pallescens

Polistichum spinulosum

Luzula campestris
\end{abstract}

Ce sont les espèces campagnes que l'on n'a pu, pour différentes raisons, intégrer à aucun groupe à signification écologique particulière.

La présence simultanée de deux ou plusieurs de ces groupes permet de caractériser un certain type de station : le tableau en définit six principaux et deux variantes (ou faciès).

\section{4. - Discussion}

Les graphiques des figures 3 et 4 permettent de se faire une idée de la distribution des espèces dans les plans $1 \times 4$ et $2 \times 3$. Nous avons vu $(\S 2.2)$ que ces axes factoriels ont une interprétation qui les rapproche. Sur ces graphiques, nous avons fait figurer les espèces « sorties » à l'analyse et, accompagnées d'un symbole pour chaque groupe, les espèces présentes dans le tableau. On voit que sur le graphique 3 , il n'y a 


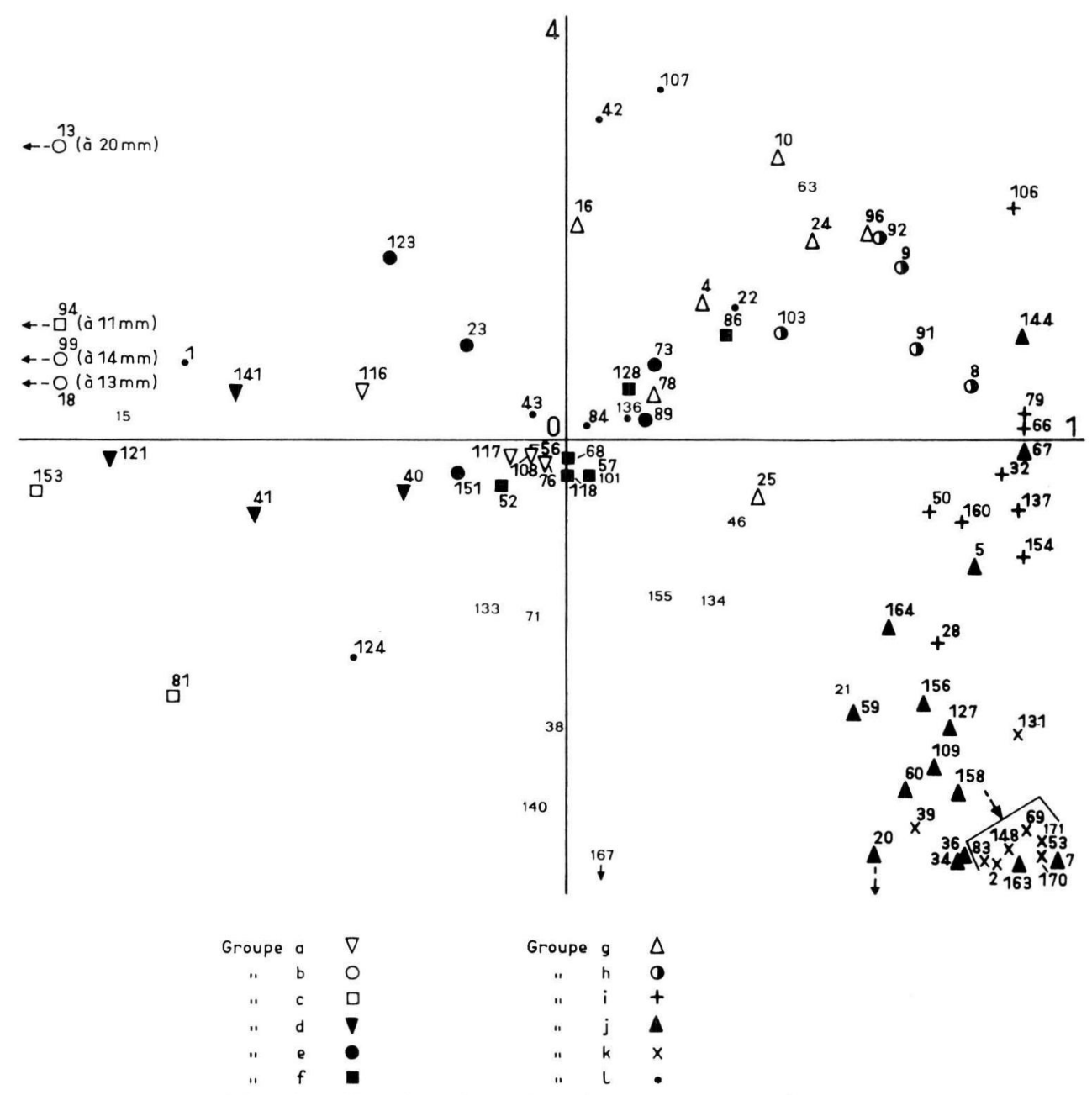

FIG. 3. - Répartition des espèces dans le plan factoriel $1 \times 4$.

Species disposition through factorial plan $1 \times 4$.

jamais de coupures nettes entre les groupes : Ils s'individualisent assez bien le long de l'axe 1 pour les groupes b à h, le long de l'axe 4 pour les groupes h à k. Le groupe a, présent partout, a ses espèces proches du zéro. Par contre la figure 5 ne donne pas du tout la même image. Ces axes traduisant la lumière, on observe une dispersion très importante des espèces de chaque groupe, sauf peut être pour celles qui présentent une tendance à la sciaphilie (f).

Les graphiques des figures 5 et 6 représentent, dans les mêmes plans, la dispersion de l'ensemble des cent soixante-dix-sept relevés. Là encore on observe (fig. 5) un bon regroupement des relevés dans le plan $1 \times 4$, la séparation entre les types 1 à 4 se faisant selon l'axe 1, la séparation de 5 et 6 selon l'axe 4. Par contre (fig. 6), on n'observe aucun regroupement dans le plan $2 \times 3$, sauf peut-être pour le type 1 , mais l'on sait par ailleurs que les stations du type 1 sont caractérisées par des espèces acidiphiles ef héliophiles. 


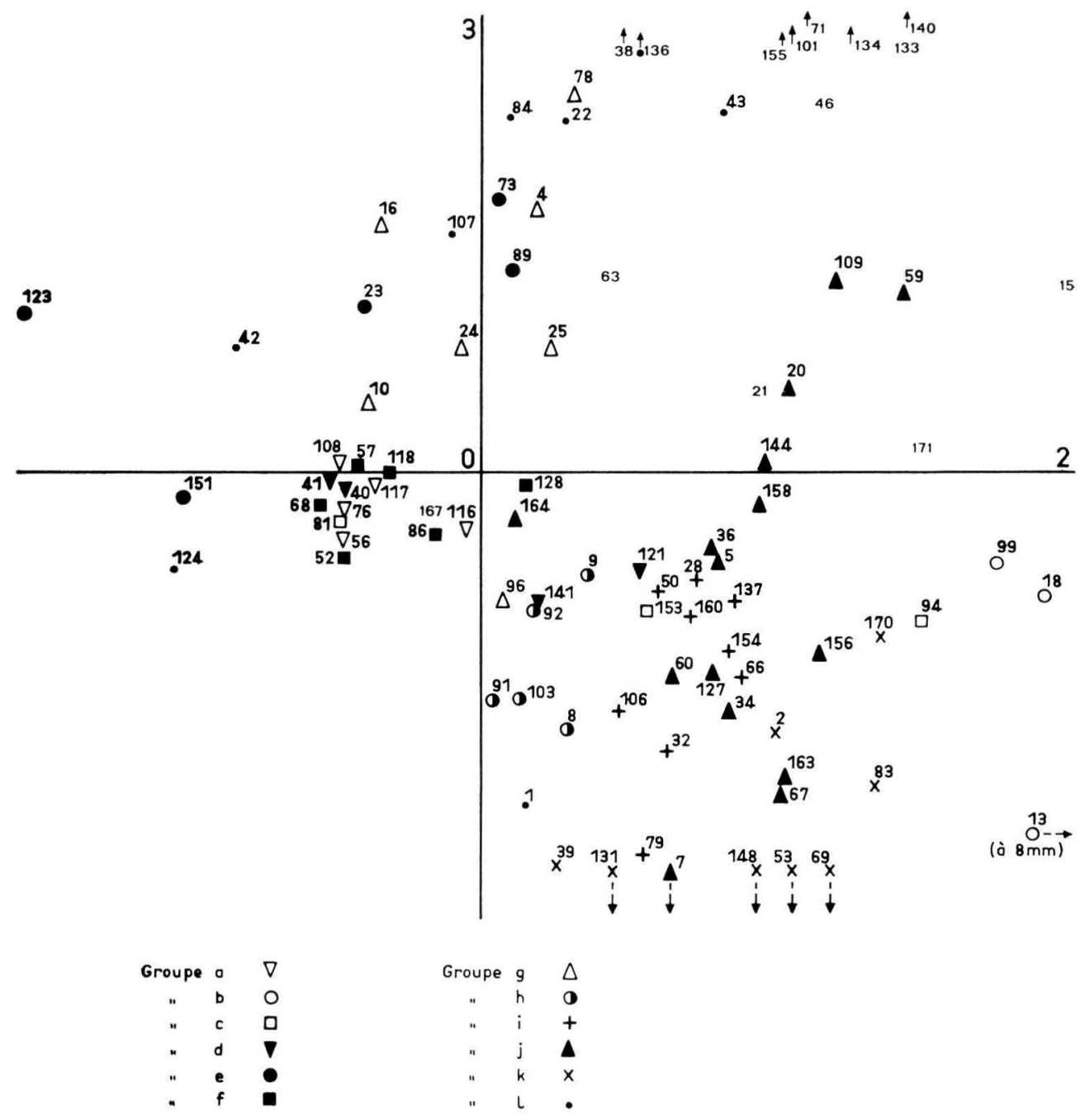

FIG. 4. - Répartition des espèces dans le plan factoriel $2 \times 3$.

Species disposition through factorial plan $2 \times 3$.

On voit également que la limite entre groupes n'est jamais tranchée, et qu'ils se chevauchent par le biais de plusieurs relevés, mais qu'il n'y a jamais de hiatus grave dans ces groupes de relevés. En fait, ceci provient de la part de subjectivité inévitable qui intervient au moment du choix de l'appartenance d'un relevé à un type ou à un autre. Soit que le relevé manque d'homogénéité, soit qu'il présente des conditions écologiques intermédiaires (qui se reflètent dans sa composition spécifique), il nous faut trancher, et c'est là qu'intervient le « métier » du biologiste. II peut en effet accorder plus d'importance à l'absence d'une espèce qu'à l'abondance d'une autre. Mais le plus important est certainement l'absence de contradiction fondamentale entre l'ordination de l'analyse des correspondances et le classement du tableau. 


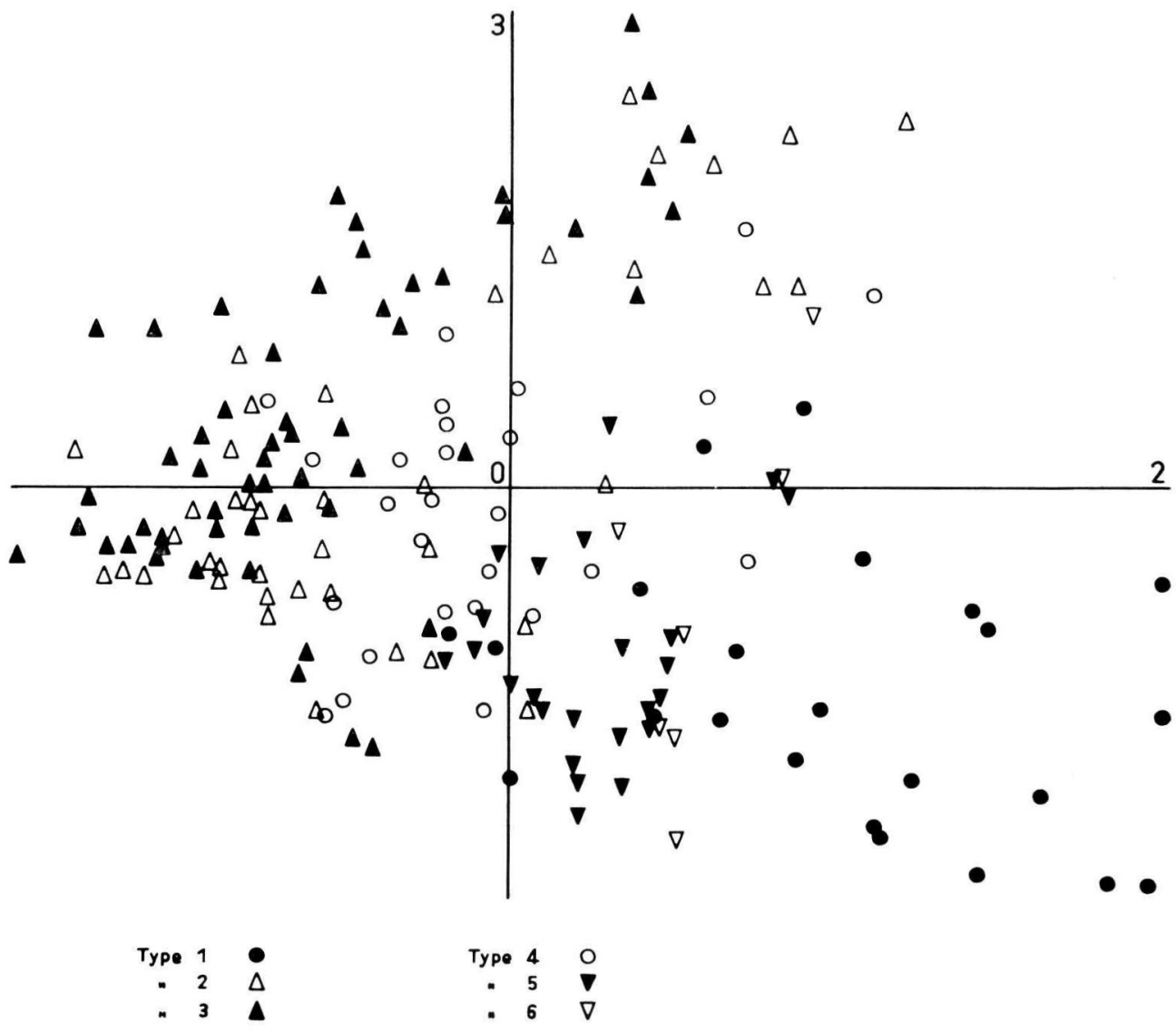

FIG. 6. - Répartition des relevés dans le plan factoriel $2 \times 3$.

Species lists disposition through factorial plan $2 \times 3$.

tation et, ce que nous n'avons pas fait mais qui est apparu plus tard comme une lacune, on note un certain nombre de données écologiques simples (concernant essentiellement le sol) qui faciliteront l'interprétation des axes factoriels ;

- un deuxième temps d'analyse de ces données qui comprend deux phases:

- une phase de « débroussaillage » au moyen de l'analyse factorielle des correspondances : cette analyse permet de définir les principaux gradients écologiques et donne un classement provisoire des relevés et des espèces,

- une phase d'élaboration du résultat final (tableau phytosociologique) qui permet d'arriver au classement des relevés en types de stations et des espèces en groupes,

- un troisième temps, dont l'importance peut varier d'une étude à l'autre, de caractérisation écologique détaillée des stations et, éventuellement, de cartographie de ces stations $\left({ }^{1}\right)$.

( $\left.{ }^{1}\right)$ Nous réservons à un autre article (Revue Forestière française $n^{\circ}$ 6-1978) la description plus précise floristique et édaphique - des stations mises en évidence par le traitement exposé ci-dessus. Y seront également exposées les liaisons station-production. 


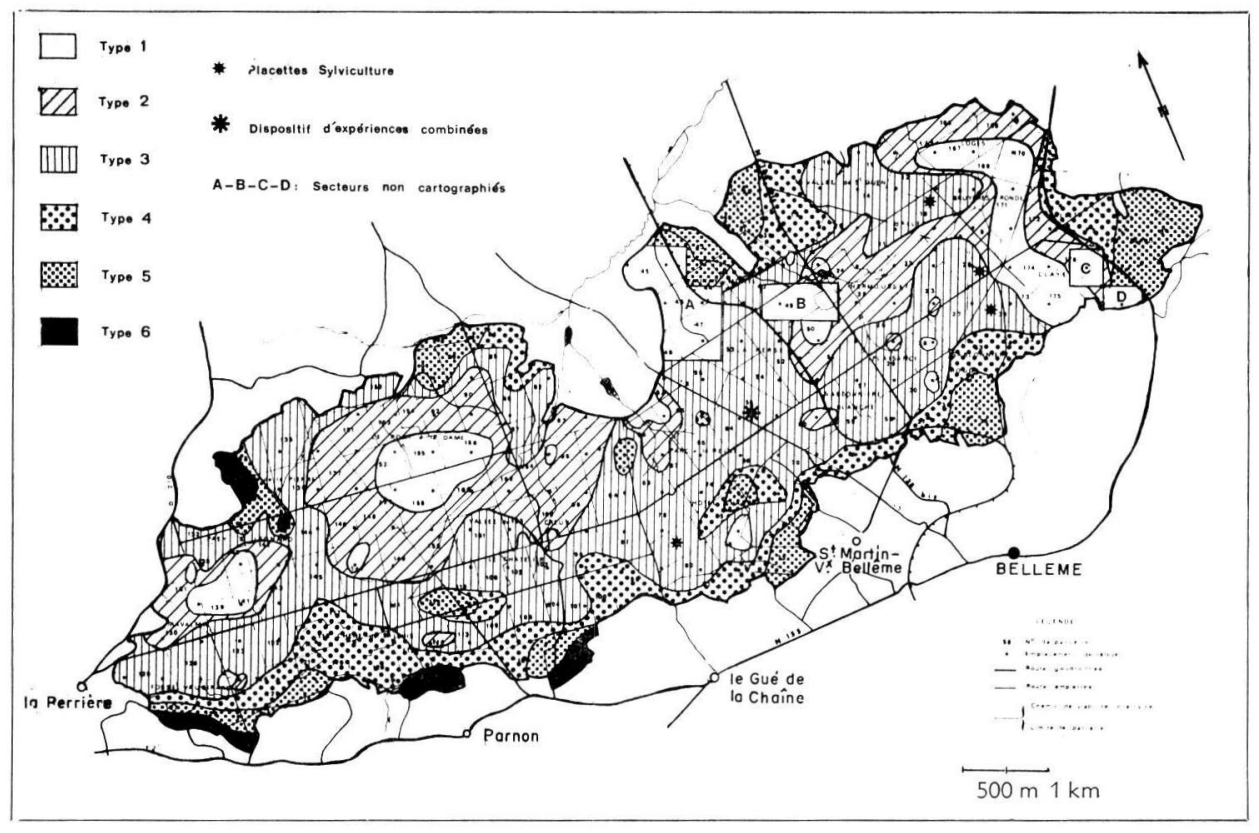

FIG. 7. - Carte schématique des stations.

Schematic nap of site types.

Cette démarche que nous proposons se présente ainsi comme un moyen terme entre une approche strictement classificatoire et une approche ordinatoire (continuum). Nous pensons en effet que la première, qui évite les zones de transition, ne permet de trancher facilement que lorsque les situations sont claires et que la seconde, en refusant d'établir des limites, ne répond pas à un besoin ultérieur de cartographie.

Terminons avec cette phrase de Dale et Anderson (1972) «It seems more likely that numerical and traditional approaches can usefully combine their better features rather than attempt to assert unilateral dominante in the field of phytosociological methodology » qui, à l'époque entrevoyaient qu'une méthode faisant la synthèse des deux approches serait un outil plus performant. II semble bien que l'école russe de Ramensky s'orientait égale ment dans cette voie. Enfin, gardons toujours à l'esprit cette mise en garde d'Austin : «Personally I would say progress will come from mathematical treatment of vegetation data which is compatible with and equivalent to our ecological understanding ».

Reçu pour publication en novembre 1978.

\section{Summary}

A method of sites classification : application to the state forest of Bellême

The purpose of this study is to present a method to define forest sites from the example of the state forest of Bellême (Orne). 
It has four parts :

- The first part shortly presents the forest (topography - main geological features - climate) and the field work. Two field trips are needed : the first one to collect phytosociological data; the second one (when the botanical characteristicts of each forest site are known) to precisely describe them from a choosen example.

- The second part refers to the office work : the data collected on the field have been analysed by the mean of the Factor Analysis of Correspondances (FAC). In this part, it is shown how the species and the species list («Relevés » in French) are sorted through the FAC. Each axis issued from the analysis is characterised each time by two groups of species (one on the positive side, the other on the negative side). An ecological signification is thereby given to the axes and points out the mains gradients. Figures 1 show how the species are grouped in plan $1 \times 2$ and figure 2 how the species lists are grouped in the same plan.

The species have been represented with an identical symbol for each of the groups defined in chapter 3.2 ; the species lists with an identical symbol for each of the sites types defined in the phytosociological table.

- The third part is divided in two chapters.

- the first one presents the method used to elaborate the phytosociological table (table 2),

- in the second one, we describe the main sociological groups defined in the table.

- The fourth part discuss, through the figures 3, 4, 5 and 6, the complementary of the two staples: FAC and phytosociological table. It is conclude that there is no contradiction between this two staples.

As a conclusion, it seems that the presented method is efficient but it would be better, during the first survey, to collect a number of simple ecological variables along with botanical data. At last, an AUSTIN's hint to the reader is recalled " progress will come from mathematical trealment of vegetation data which is compatible with and equivalent to our ecological understanting ".

\section{Références bibliographiques}

AUSSENAC G., 1973. Rapport préliminaire, Recherche sur la régénération du Chêne et du Hêtre, Etude climatologique. Document ronéo, C.N.R.F.

AUSTIN M. P., 1971. Models and analysis of descriptive vegetation data. In "Mathematical models in ecology ». Blackwell Scientific publications, 1972, 398 p.

AUSTIN M. P., ORLOCI L., 1966. Geometrics models in ecology. II. - An evalutation of some ordination techniques. Journal of Ecology, 54, pp. 217-227.

BACHACOU J., 1973. L'effet Guttman dans l'analyse de données phyłosociologiques. Document ronéo, Station de Biométrie, C.N.R.F.

BACHACOU J., 1975. L'analyse des correspondances, Document ronéo, C.N.R.F.

BACHACOU J., 1976. Analyse en composantes principales. Document ronéo, Station de Biométrie, C.N.R.F., no 76-04.

BECKER M., 1969. Le Hêtre (Fagus silvatica L.) et ses problèmes en forêt de Villers-Cotterêts (Aisne). Contribution à la mise au point d'une méthode dynamique d'étude écologique du milieu forestier. Ann. Sci. forest., 26 (2), 141-182.

BECKER M., 1971. Etude des relations sol-végétation, en conditions d'hydromorphie dans une forêt de la plaine lorraine. Thèse d'Etat de l'Université de Nancy.

BENZECRI J. P. et al., 1973. L'analyse des données (2 tomes). — Dunod.

BOTTLIKOVA A. et al., 1976. Quelques résultats obtenus par l'analyse factorielle et les profils écologiques sur des observations phyto-écologiques recueillies de la vallée de Liptov (Tchécoslovaquie). Vegetatio, vol. 31, 2 ; 79-91.

COETZEE B. J., WERGER M. J. A., 1975. On association analysis and the classification of plant communities. Vegetatio, vol. 30, $3 ; 201-206$.

CURTIS J. J., 1959. The vegetation of Wisconsin : an ordination of plant communities. Univ. of Wisconsin press, Madison, 657 p.

CZEKANOWSKI J., 1909. Zur differentialdiagnose der Neanderthalgruppe. Korresponenzblatt der deutschen Gesellschaft für anthropologie 40, pp. 44-47.

DAGNELIE P., 1960. Contribution à l'étude des communautés végétales par l'analyse factorielle. Bull. Serv. Carte phytogéog., B, 5, 1-195.

DAGNELIE P. L'analyse factorielle. Document ronéo de la Faculté des Sciences agronomiques de l'Etat, Gembloux-Belgique.

DALE M. B., 1974. On objectives of methods of ordination. Vegetatio, 30, 1, 15-32.

DALE M. B., ANDERSON D. J., 1972. Qualitative and quantitative information analysis. Journal of Ecology, 60, 639-654. 
DIDAY E., LEBART L., 1977. L'analyse des données. La Recherche, no 74, janv. 1977, p. 15-25.

ESTEVE J., 1978. Les méthodes d'ordination : éléments pour une discussion in Biométrie et écologie, $\mathrm{n}^{\circ}$ 1, I.N.R.A. Dpmt. de Biométrie.

GAUCH H. G., WHITTAKER R. H., 1972. Comparison of ordination techniques, Ecology, 53 p., 868875.

GAUCH H. G., WHITTAKER R. H., 1976. Simulation of community patterns, Vegetatio, vol. 33, 1 : 13-16.

GOUNOT M., 1969. Méthodes d'éfude quantitative de la végétation. Masson et Cie, 314 p.

GRANT-COTTAM, GLENN GOFF L., WHITTAKER R. H., 1973. Wisconsin comparative ordination in Handbook of vegetation science, Pp. 193-221.

GRIEG-SMITH P., 1964. Quantitative plant ecology. 2nd Ed. Butterworth inc., Washington D. C., 256 p. GUINOCHET M., 1973. Phytosociologie, Masson et Cie, Paris, 227 p.

KESSEL S. R., WITTAKER R. H., 1976. Comparisons of three ordination techniques. Vegetatio, vol. 32 , $1: 21-29$.

KSHIRSAGAR A. M., 1972. Multivariate analysis, Marcel Deleker, 1972.

LACOSTE A., 1972. La végétation de l'étage subalpin du bassin supérieur de la Tinée (A. M.). Application de l'analyse multidimensionnelle aux données floristiques, publié par Phyfo-coenologia, vol. $3,1975,88+345+32$ p.

LACOSTE A., ROUX M., 1971. L'analyse multidimensionnelle en phytosociologie et écologie. I. - L'analyse des données floristiques. Oecologia plantarum 6, pp. 353-369.

LACOSTE A., ROUX M., 1972. L'analyse multidimensionnelle en phytosociologie et en Ecologie. II. - L'analyse des données écologiques et l'analyse globale. Oecologia plantarum, 7, pp. 125146.

MAC INTOSH R. P., 1967. The continuum concept of vegetation. Bot. Rev., 33, pp. 130-137.

MENAR M., BELANGER J., 1976. Etude des stations entre les caractères du milieu et la production forestière par l'analyse factorielle des correspondances. Serv. de la Rech. Gen. des forêts - Min. des terres et forêts du Québec, Mémoire no 24.

NOY-MEIER I., 1974. Catenation : quantitative methods for the definition of coenoclines. Vegetatio, 29, PP. 89-99.

ORLOCI L., 1966. Geometrics models in ecology. I. - The theory and application of some ordination methods. Journal of Ecology, 54, pp. 193-215.

ORLOCI L., 1975. Multivariate analysis in vegetation research. Dr W. Junk bv Publishers, 276 p.

PICARD J. F., 1970. Les forêts sur Rhétien dans le département des Vosges. Nouvelle contribution à la mise au point d'une méthode dynamique d'étude phyto-écologique du milieu forestier. Thèse de $3^{\mathrm{e}}$ cycle de l'Université de Nancy.

ROMANE F., 1977. Application à la phyto-écologie de quelques méthodes d'analyse multivariable. Thèse de docteur-ingénieur, Université de Montpellier, $\mathrm{n}^{\circ}$ AO 5865, $110 \mathrm{p}$.

ROUX G., ROUX M., 1973. A propos de quelques méthodes de classification en phytosociologie. In Benzecri, L'analyse des données, t. 1 : La taxinomie, $615 \mathrm{p}$.

SOBOLEV L. N., UTEKHIN V. D., 1973. Russian (Ramensky) Approaches to Community Systematization in Handbook of vegetation science, edited by R. M. WHITTAKER, 737 p.

SWAN J. M. A., 1970. An examination of some ordinating problems, by use of simulated data. Ecology, 51, pp. 89-101.

SWAN J. M. A., DIX R. L., 1966. The phytosociological structure of upland forest ad Candle Lake, Saskatchewan. journal Ecol., 54, pp. 13-40.

WHITTAKER R. H., 1960. Vegetation of the Siskiyou mountains, Oregon and California. Ecol. monogr. 30, pp. 279-338.

WHITTAKER R. H., 1967. Gradient analysis of vegetation, Biol. rev., 42, pp. 207-264.

WHITTAKER R. H., 1973. Handbook of vegetation science. Edited by R. H. WHITTAKER, 757 p. 